\title{
Duplication cyst of the cecum: A case report
}

\author{
Akash Pati · Hiranya Kishore Mohanty · Pravas Chandra Subudhi · Ramnarayan Dash · Pramod Kumar Mohanty • \\ Rabindra Kumar Mahapatra
}

Received: 17 December 2008 / Accepted: 12 February 2010

(C) Association of Surgeons of India 2009

\begin{abstract}
Duplication of alimentary tract is one of the rare congenital anomalies. Acase of duplication cyst of the cecum, presented in the 3rd month of life, as intestinal obstruction. Excision of the cyst along with cecum and appendix was done. The child had an uneventful postoperative recovery following ileoascending anastomosis.
\end{abstract}

Keywords Caecal duplication cyst $\cdot$ Intestinal obstruction
A $2 \frac{1}{2}$ months old female child presented to the emergency department with features of intestinal obstruction for the last 3 days. The baby was moderately dehydrated. Upright skiagram of abdomen confirmed the diagnosis of intestinal obstruction. Ultrasonogram revealed a cystic mass in the right iliac fossa. Peroperatively a cystic mass was visible adjacent to the cecum which was compressing the cecum from outside. The cyst was present on the mesenteric side of the cecum and was sharing a common wall with it. The wall of the cecum was opened. It was seen that the cyst has caused obstruction by projecting into the lumen of the cecum and obliterating the lumen.

The terminal ileum, cecum, appendix and the ascending colon were excised along with the cyst. End-to-end anastomosis of the bowel was done. On biopsy there were goblet cells in the mucosa characteristic of large intestine. The cyst had all the layers of the intestine and was sharing a common wall with the cecum which confirmed it to be duplication cyst of the cecum. Patient had a smooth uneventful postoperative recovery. On follow up after 3 months the patient is doing well.

Duplications have been associated with congenital malformations of skeletal, gastrointestinal, or genitourinary tract however there was no associated malformation in the present case.

Small intestine, being the most common site, duplications of the cecum is rare. Incidence of duplication of cecum alone is difficult to find from literature. Caecal duplication cyst presenting as intussusception has been reported by some authors [1,2]. Ratan et al. [3] have reported a case of intestinal obstruction in a neonate caused by caecal duplication cyst. Ultrasonogram is the most common imaging modality used to image duplication cyst. It has the added benefit of detecting genitourinary anomalies in case of colonic duplication. At ultrasonogram the duplication cysts

A. Pati $(\bowtie)$

E-mail: patiakash@rediffmail.com 
demonstrate an echogenic inner mucosal layer and hyper echoic outer muscular layer. Technetium pertechnetate scan can be used to pick up the ectopic gastric mucosa present in the duplication cyst. The preferred treatment is by resecting the duplication and its attached normal colon.

Duplications of the intestine are rare. The biggest series one of only 67 cases was published by Gross. The collection of cases took nearly 30 years. Half of the duplication occurs in the ileum making cecum a very rare site for duplication cysts to occur.

\section{References}

1. Rizalar R, Somuncu S, Sozubir S, Yildiz L, Gurses N (1996) Caecal duplications: a rare cause for secondary intussusception, Indian J Pediatr 63(4):563-566

2. Kum CK, Prabhakaran K, Lee YS, Fok E (1991) Cystic duplication of the cecum mimicking intussusception $\mathrm{J}$ Singapore Paediatr Soc 33(1-2):37-39

3. Ratan SK, Kulsreshtha R, Ratan J (2001) Cystic duplication of the cecum with segmental dilatation of the ileum: report of a case. Surg Today 31(1):72-75 